\title{
How to perform an efficient learning process for a combined POD and PGD soil urban thermal model?
}

\author{
Marie-Hélène AZAM ${ }^{2,1}$, Sihem GUERNOUTI ${ }^{2,1}$, Marjorie MUSY ${ }^{2,1}$, Philippe POULLAIN ${ }^{1}$ \\ ${ }^{1}$ Univ. Nantes, CNRS UMR 6183, GeM, F-44000 Nantes, France \\ ${ }^{2}$ Cerema, Equipe-Projet BPE, F-44000 Nantes, France
}

\begin{abstract}
A parametric soil model has been developed to improve the computational time of microclimate simulation tools. It combines the use of two methods: the Proper Generalized Decomposition and the Proper Orthogonal Decomposition. Offline, a learning process is required to build the model, before its use online. A methodology to select a short and representative learning process needs to be developed. The $\mathrm{k}$-means clustering method is used to build a training climate made of 24 days representative of a full climate. The offline computation costs are reduced by $94.4 \%$ for an error of $0.8 \%$.
\end{abstract}

\section{Introduction}

In the global warming context, urban densification has negative consequences for the local city climate. It leads to the reinforcement of the Urban Heat Island (UHI) phenomenon. That is why, the development of cities must now be carried out considering the UHI as an environmental issue, since it has consequences for the health and comfort of occupants as well as on the energy consumption of buildings.

The storage of solar heat flux by urban materials during day and its release at night-time is one of the main causes of UHI (Oke, 2002). This heat flux is greater in urban areas than in rural ones due to the higher inertia of the urban construction materials. The surface temperatures drive the different heat fluxes (convection, conduction, and long wave radiative exchanges) at the urban surface. For this reason, it is of major importance to assess these variables of the urban microclimate.

In order to quantify the impact of mitigation solutions of UHI on local microclimate, several numerical models have been developed (Mirzaei, 2015). Most of the microclimate tools combine several one-dimensional thermal models (soil, buildings, etc.) using a cosimulation approach. The urban scene is meshed and for each cell of the mesh, the urban soil thermal model intends to compute the surface energy balance.

At each coupling time step, the transient heat transfer equation needs to be solved. It computes the temperature profile inside the soil that depends on the boundary conditions calculated by the urban microclimate tool. Every possible combination of boundary conditions defines a set of parameters. The heat equation needs then to be solved for each set of boundary conditions. To reduce the computational time, a reduced parametric model has been proposed in Azam et al. (2018). It combines the use of the Proper Generalized Decomposition (PGD) method to generate a parametric solution of the previously described problem and the use of the Proper Orthogonal Decomposition (POD) to reduce the number of parameters involved in the parametric PGD model.

The studied parametric urban soil model is a combination of two reduced order model (ROM) techniques: an a priori method (PGD) and an a posteriori one (POD). For a posteriori model order reduction method, a learning process, done offline, is required. This learning period should be as short as possible and representative of all cases that will be simulated online, to obtain an efficient combined parametric model. In the literature, the POD method has mainly been applied to fluid dynamics, few applications focus on heat transfers and even fewer on buildings or urban thermal modeling. A methodology to select a short and representative learning process needs to be developed for our purposes.

In this paper, we propose to investigate the use of clustering method to reduce the necessary training period of the POD basis. A methodology is proposed for selecting the most representative data from a full training climate. The obtained training climate is then evaluated on the coupled model behavior within a complete urban heat balance.

\section{Methods}

\section{Physical problem and LOM}

The studied physical problem involves transient onedimensional heat conduction through a soil column (Equation 1) for time interval $\Omega_{t}$ such that $t \in[0, \tau]$ and space interval $\Omega_{x}$ such that $x \in[0, L]$ :

$$
c_{i} \frac{\partial T_{i}}{\partial t}=\frac{\partial}{\partial x}\left(k_{i} \frac{\partial T_{i}}{\partial x}\right) i=1,2, \ldots, I
$$

where $k$ is the thermal conductivity and $c$ the specific heat capacity, constant over time. Both these variables depend on the space variable $x$ since the domain is made of several layers $i$. At the soil surface $(x=0)$, a RoBin boundary condition is assumed:

$$
-k_{1} \frac{\partial T}{\partial x}=q_{n e t}-h\left(T-T_{a}\right)
$$


where $q_{\text {net }}$ is the net radiative heat flux. This flux is calculated from the balance of short wave (SW) $q_{S W}$ and long wave (LW) $q_{L W}$ radiative heat fluxes exchanged between the urban surface and the surrounding surfaces. The sensible heat flux is calculated from the air temperature $T_{a}$ and from a convective heat transfer coefficient $h$. As each flux varies over time, the boundary condition is not constant over time. At the bottom $x=L$, the soil temperature is imposed through a DIRICHLET boundary condition:

$$
T=T_{\infty}
$$

where $T_{\infty}$ is a constant daily temperature. At $t=0$, the initial temperature is set using a space temperature profile:

$$
T=T_{0}(x)
$$

The contact between two layers $i$ and $i+1$ is assumed perfect. That means that the continuity in temperature and heat flux at the interface between is ensured. The previous equation (1) can be written in a dimensionless form as:

$$
c_{i}^{\star} \frac{\partial T^{\star}}{\partial t^{\star}}=F_{o} \frac{\partial}{\partial x^{\star}}\left(k_{i}^{\star} \frac{\partial T^{\star}}{\partial x^{\star}}\right),
$$

for $t^{\star} \in[0, \Gamma]$ and space interval $x^{\star} \in[0,1]$. The boundary conditions can be written as follows:

$$
\begin{gathered}
k_{1}^{\star} \frac{\partial T^{\star}}{\partial x^{\star}}=B i_{o}\left(T^{\star}-T_{a}^{\star}\right)-q_{\text {net }}^{\star}, \text { at } x^{\star}=0 \\
T^{\star}=T_{\infty}^{\star}, \text { at } x^{\star}=1 \\
T^{\star}=0, \text { at } t^{\star}=0
\end{gathered}
$$

where the dimensionless quantities are defined as:

$x^{\star}=\frac{x}{L} ; \quad T^{\star}=\frac{T-T_{0}}{T_{0}} ; \quad t^{\star}=\frac{t}{t_{\text {ref }}} ; \quad k_{i}^{\star}=\frac{k_{i}}{k_{1}} ;$

$c_{i}^{\star}=\frac{c_{i}}{c_{1}} ; \quad B i=\frac{h . L}{k_{1}} ; \quad F o=\frac{k_{1} \cdot t_{r e f}}{c_{1} L^{2}}=1$

$t_{\text {ref }}=\frac{c_{1} L^{2}}{k_{1}} ; \quad T_{a}^{\star}=-1+\frac{T_{a}}{T_{0}} ; \quad q_{\text {net }}^{\star}=\frac{q_{\text {net }} . L}{k_{1} \cdot T_{0}} ;$

$T_{\infty}^{\star}:=\frac{T_{\infty}-T_{0}}{T_{0}} ; \quad \Gamma=\frac{\tau}{t_{\text {ref }}}$

For the sake of notation compactness, the $\star$ is not mentioned in the following equations. Only the dimensionless equations and values are used.

The defined problem can be solved using any classic numerical method. In this paper, the so-called Large Original Model (LOM) is defined as the solution of the previous problem using the finite difference method. The spatial domain $\Omega_{x}$ is discretized into a grid composed of Nx nodes, while the time domain $\Omega_{t}$ is discretized into Nt time steps. An implicit scheme is employed, with backward first-order derivatives for the time derivation and a center second-order derivative scheme for the spatial derivation.

\section{Parametric problem}

As described in Azam et al. (2018), the soil model is combined with several one-dimensional models using a co-simulation approach to assess the thermal behavior of an urban scene. During a time interval $t \in\left[t^{n}, t^{n+1}\right]$, each model computes the field of interest, which consists of the temperature of each surface. Particularly, the soil model computes the 1-D heat transfer equation for each cell of the scene mesh. Given the temperature profile at time step $t^{n}$ and the boundary conditions at time step $t^{n+1}$, the model calculates the temperature profile at time step $t^{n+1}$. The previous temperature profile at $t^{n}$ can be seen as an initial condition of the problem solved between $t^{n}$ and $t^{n+1}$. Every possible combination of boundary conditions and initial condition could then define sets of parameters. The heat equation needs then to be solved for all of these sets of parameters. To reduce the computational time, a parametric model could be defined, and written explicitly. The implicit semi-discretization in time of Equation 5 yields to:

$$
T^{n+1}=T^{n}+d t \frac{\partial^{2} T^{n+1}}{\partial x^{2}}
$$

with the previously described boundary conditions.

\section{A combined PGD+POD parametric ROM}

The Proper Generalized Decomposition method is used in order to propose an accurate parametric solution of the formulated heat transfer problem. To achieve a generic solution to this problem, the model boundary conditions and initial conditions need to be defined as parameters. This means that the PGD parametric ROM must be calculated for any boundary condition and initial condition over a predefined interval. The model can be developed as a space (x) - boundary conditions (BC) - initial condition (IC) separation of the solution.

The challenge therefore is to compute a parametric model for the field $T^{n+1}$ by solving equation 9 in searching for a separate solution as follows:

$$
T^{n+1}=\sum_{m=1}^{\mathcal{M}} X_{m}(x) Q_{m}\left(q^{n+1}\right) F_{m}\left(T^{n}\right)
$$

with $\mathcal{M}$ the number of PGD modes in the approximation of the solution.

As presented above, the parametric formulation of the problem requires taking the previous time step temperature profile into account as a parameter. Once discretized in space, the temperature profile of the initial condition is no longer of infinite dimension: the description of the previous temperature profile provides one piece of information per node. It implies inputting as many parameters in the PGD parametric model as the number of nodes in the discretization grid, plus the $\mathrm{BC}$ and the spatial coordinates. To avoid this large number of parameters, the initial condition needs to be parameterized. For that purpose, we propose to approximate the initial temperature field, by its projection on a reduced-order basis, as follows:

$$
T^{n}=\sum_{j=1}^{\mathcal{N}} \zeta_{i} \Phi_{i}
$$

with $\Phi$ the approximation reduced-order basis, $\mathcal{N}$ the number of modes in the reduced-order basis and $\zeta_{i}$ coefficients of the temperature field projection on the 
basis.

Several approximation basis can be used to parameterized the initial condition. The use of the Proper Orthogonal Decomposition (POD) has shown good results in González et al. (2012).

The POD method consists in searching for a set of basis functions $\Phi$ that approximates the temperature profile $T^{n}$ from the eigenvalues and eigenmodes. In order to build the POD basis, a collection of snapshots is needed. In the snapshot matrix, each column represents a snapshot of the temperature profile at a given time step. This matrix defines the terms of the learning process. It has an impact on the performance of the reduced basis. For this reason, the snapshots must be representative of the problem (boundary values, initial conditions, materials properties).

For more details on the development of the specific combined parametric model, readers can see Azam et al. (2018). A complete description of the POD and PGD method can be found in Chinesta et al. (2013).

\section{Offline and online Strategy}

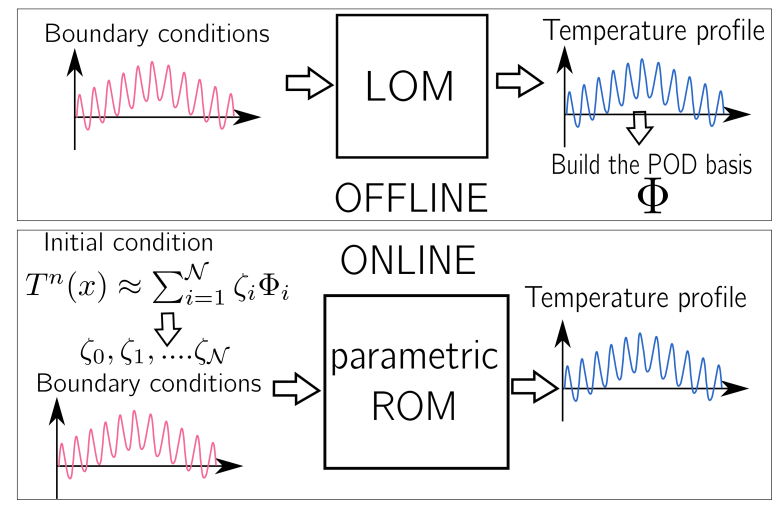

Figure 1: Offline and Online strategy.

The combined parametric soil model features an offline/online strategy. All steps are detailed in figure 1. The POD method is used to parameterized the temperature profile inside the soil at the previous time step. In order to perform this configuration, we first need to build a POD basis from a set of snapshots calculated by the LOM (Finite Difference method). This basis is computed once offline. A minimum number of modes in the POD basis, $\mathcal{N}$, is defined to achieve the targeted accuracy. Note that the number of modes has a direct influence on the number of parameters used in the PGD model. The parametric model can then be solved with the PGD algorithm for a spatial mesh, a time interval of $\left(t^{n}\right.$, $\left.t^{n+1}\right)$, any value of the boundary condition defined within a discretized interval and for each mode of the POD basis. Once the PGD parametric model has been built, it can be applied online for any value within the previously defined intervals. In the online stage, a small algebraic system of equations needs to be solved to compute the coefficients of the parameterized initial condition. Afterwords, the evaluation of the solution requires no more than reading a lookup table (the PGD parametric model).
Learning process strategies

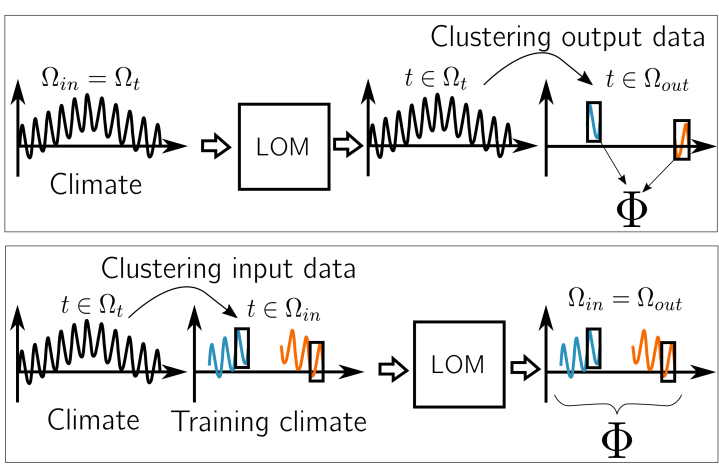

Figure 2: Methodologies to reduce the training period of the POD basis.

The POD method is part of the a posteriori ROM, as it is an approach based on learning from available data. In order to build the POD basis, a set of snapshots calculated by the LOM is needed. This consists in carrying out simulations for different parameter samples: boundary condition for example. Data collected in the learning stage are used to construct a reduced basis. The latter is assumed to span the entire solution space for any parameters. The previously defined offline/online strategy can be very time saving, however questions remain. As presented by Borzacchiello et al. (2017) one of the various questions related to this type of methods is the sampling. Offline sampling is very important as it will determine the model accuracy. It has to be fine enough to ensure that the dynamics is captured within the level of desired accuracy. On the other hand, extensive sampling is costly in terms of computational time. Therefore, to obtain an efficient combined parametric model, this learning period should be as short as possible and representative of all the cases that will be simulated online. The selection of an efficient learning period is then a new challenge to ensure model efficiency.

In our field of study, we generally need to sample climate data (boundary conditions). Several strategies can be used to build a training climate as short as possible. Two approaches stand out: one focuses on the output data of the model, the second one on the input data (figure 2).

In the first approach, the LOM is run for the entire climate data period $\Omega_{t}$, but the POD basis is built only on a part (few typical days) of the output data (upper part of the figure 2). Those days are selected for their characteristics, and the learning process is reduced to a training period $\Omega_{\text {out }}$, which is shorter than $\Omega_{t}$.

In the second approach, the LOM is run for a period made of the most important days (lower part of the figure 2). This period of simulation $\Omega_{i n}$ is shorter than $\Omega_{t}$. The POD basis is thereby built on these results.

In order to clarify the vocabulary used below, we dissociate the terminology used for both approaches : 
clustering the output data set to reduce the training period of the POD basis; clustering the input data set to reduce the training climate period.

The development of a training climate has the main advantage to reduce the training period of the POD basis directly. However, when clustering the output, other variables than the boundary conditions can be used to define the training data set as the surface temperature for example. The main drawback of this approach is the fact that the LOM needs to be run for the whole data period $\Omega_{t}$.

Several methods have been proposed to define a training climate. In the POD framework, applied to thermal heat transfer in building envelopes, Berger et al. (2018) proposed a training climate made of daily sequences of the input data. This choice is based on spectrum analysis of climate data: the period of $24 \mathrm{~h}$ represents $10 \%$ of the total energy spectrum of the year while a $12 \mathrm{~h}$ period would only represent $1.5 \%$. They have tested several training climates, selecting the days based on daily average values of air temperature or equivalent air temperature to consider radiative heat fluxes. The sequences are made of extremum days to train the model with limit values of the interval. For the POD method, 2 days are sufficient to reach the desired accuracy. Similar approaches propose to select one day per month or per season depending on the problem variation. The number of days and the way to select them are then based on the field expertise. In another context, Domínguez-Muñoz et al. (2011) and Fazlollahi et al. (2014) proposed to use a statistical method to design the training climate with clustering analysis method. The main idea is to quantify how different two days are according to a parameter. Based on their mutual distance, days are arranged into clusters. The center day of this cluster is then defined has the most representative day. The training climate can then be made of the representative days.

In the case of dynamical problem, the solution at a time step $t^{n}$ depends on the solution at the previous time steps $t^{n-1}$ or $t^{n-2}$. The inertia should be then taken into account to get a training climate that reproduces reliable temperature profiles as if the full climate was simulated. Ribault (2017) proposed a strategy based on k-means clustering method with some specific constraints. He deleted only days that do not have an impact on the representative day and do not prejudice to the sequence continuity. The developed methodology leads to a training climate made of 83 days (instead 365 days) with a relative error of $7 \%$ on the building energy needs.

\section{K-means clustering method}

Clustering method is one of the strategies to reduce the training period of the POD basis. The aim of this method is to merge similar days into groups (clusters). Each group can then be represented by one of its members, called the centroid, which is the most

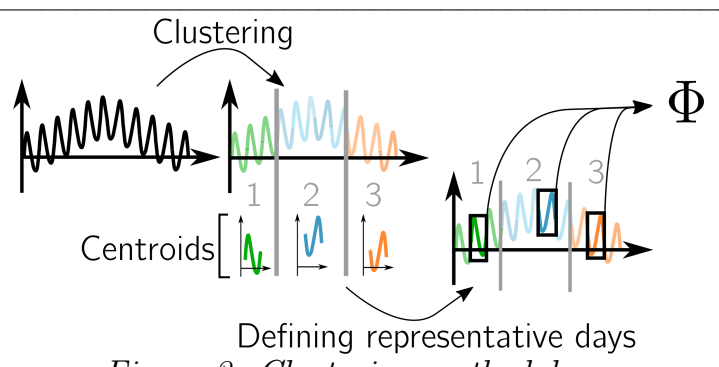

Figure 3: Clustering methodology.

centrally located member of the group. Figure 3 gives a schematic drawing of the method. To implement the k-means clustering method, the initial data set is arranged into a matrix with as many rows as the number of objects that needs to be classified and as many columns as the number of variables. If the output surface temperature is the studied variable, the matrix will be made of 365 rows (one object per day) and 24 columns (one variable per hour). The euclidean distance between two days needs then to be calculated. Each distance is arranged into a symmetric matrix called the dissimilarity matrix. It contains all the information that the clustering algorithm needs to partition the data. Many clustering algorithms have been proposed in the literature. We use k-means method and the algorithm provided by Scikit learn Python library (Pedregosa et al. (2011)) which uses Lloyd's algorithm. The algorithm is initialized by choosing the initial position of the centroids. Then samples with the smallest euclidean distance to the centroid are gathered to create a cluster. The centroid is updated according to the new mean value of all the samples in the cluster. The difference between the old and the new centroid is computed. This two last steps are repeated until the difference between the old and the new centroid reaches a threshold.

The performance of the k-means algorithm depends on two parameters: the starting point and the number of clusters. Different random starting points could be used to run the algorithm and overcome the first issue. In the Scikit learn algorithm, the centroids are chosen to be distant from each other instead of a random initialization. For the second issue, the best clustering approach is elected based on a statistical measure or on the domain expertise. Fazlollahi et al. (2014) evaluates the compactness character of the clusters and the separation between clusters. Ribault (2017) uses the final heating and cooling energy needs to evaluate the quality of its training climates.

\section{Methodology of learning period development}

The main objective of this work is the development of a methodology to select a short and representative training climate in order to reduce the training period of the POD basis. Three questions arise: (1) Is it possible to build the POD basis on a reduced training period? (2) How can the training climate be defined to obtain the desired output data set for a reduced training period? (3) What is the impact of this training climate on the overall accuracy of the model and 
offline calculation time? In order to answer the first question, a study is carried out on the reduction of the training period of the POD basis. For that purpose, the clustering method is applied on the output data set to define the number of representative days needed in the training climate. Once this first step has been completed, the clustering method is applied on the input data set to answer the second question. The use of different input variables are compared to select the representative days. The number of days needed to represent the inertia is studied. The training climate is then made of the representatives days and several others days for the inertia. Finally, in order to answer the third question, the combined model performance with the defined training climate is evaluated based on accuracy and CPU calculation time results.

\section{Assessment's indicator}

An indicator to qualify the accuracy of the POD basis is needed. The $\ell_{2}$ norm (or Root Mean Square Error) noted $\varepsilon_{2}$ is chosen. It is computed as a spatial function by the following discrete $\ell_{2}$ formulation, where $\mathrm{N}_{\mathrm{x}}$ is the number of elements in the spatial mesh.

$$
\varepsilon_{2}(t)=\sqrt{\frac{1}{\mathrm{~N}_{\mathrm{x}}} \sum_{0}^{\mathrm{N}_{\mathrm{x}}}\left[T_{r e f}(x, t)-T_{\text {proj }}(x, t)\right]^{2}}
$$

The reference solution noted $T_{r e f}(x, t)$ defines the temperature profile computed by the LOM with the full climate period $\Omega_{t}$. $T_{\text {proj }}(x, t)$ defines its projection on the POD basis $\Phi$ for a number of modes $\mathcal{N}$ (equation (11)). The global error $\varepsilon_{\infty}$ is given by the maximum of the previous function $\varepsilon_{2}(t)$. For each step, the CPU calculation are evaluated on a Macbook Pro (16Go RAM Intel Core i7 $2.3 \mathrm{GHz}$ ). The CPU calculation time are normalized by the maximum CPU time measured.

\section{Results}

\section{Description of the case study}

The developed combined model is applied to the case of a theoretical urban environment consisting of two canyon streets. This case study has already been used in Azam et al. (2018), only main information are recalled here after. For this study, the combined model is coupled with the SOLENE-microclimat simulation tool (Musy et al., 2015) through the use of the pingpong method. The microclimate tool is used to take into account the surrounding surfaces in the surface energy budget (i.e. SW and LW radiative budget). The objective here is to study the behavior of the ROM model implemented within a complete urban heat balance.

The urban scene is composed of a square and two canyon streets. Only the center of the open area is studied in this paper. The impervious soil represents $32 \%$ of the total mesh content in the urban scene. The simulation is run for a complete year, with a one-hour time step. Weather data acquired in 2010 for the city of Nantes are used as inputs.

\section{Is it possible to build the POD basis on a re- duced training period?}

The first study aims at quantifying how much the output data set can be reduced to get an accurate POD basis. For that purpose the number of temperature profiles used to build the POD basis varies from 6 to 8760 (the total number of time steps over a year). In order to evaluate the impact of the training period on the accuracy of the POD basis alone, the temperature profiles computed by the LOM are compared to their approximation for different numbers of modes $\mathcal{N}$.

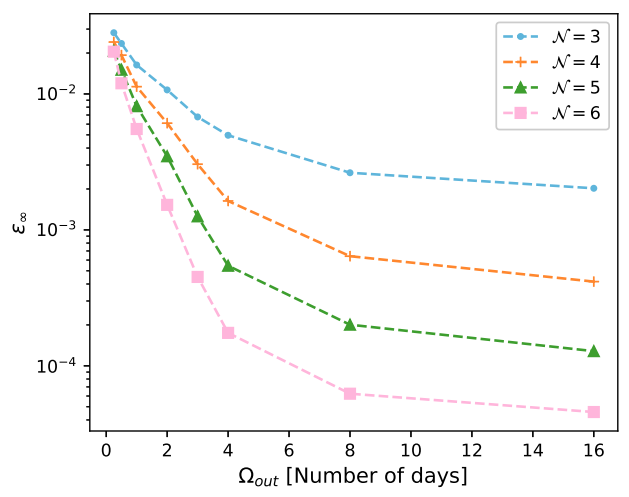

Figure 4: Impact of the training period $\Omega_{\text {out }}$ on the $P O D$ basis accuracy for various numbers of $P O D$ modes $\mathcal{N}$.

Figure 4 presents the results.For any number of modes in the POD basis, the error decreases as the number of days in the output training climate increases until a plateau is reached after 15 days. As the accuracy of the POD basis is proportional to the number of modes $\mathcal{N}$, this plateau represents the limit of accuracy of the basis. For $\mathcal{N}=5$, and $\Omega_{\text {out }}=15$, an accuracy of $\varepsilon=1.210^{-4}$ is reached. A whole year training will only improve the basis error from $10^{-5}$ for $\mathcal{N}=5$. Without applying a methodology to select the optimum number of days to train the POD basis, this first result reveals that a whole year training climate is not necessary. However, no special attention has been paid for now on the selection of the output data set.

After this first investigation, the clustering method is applied to the output data set to optimize the training period of the POD basis. The main idea is to divide the output data set into clusters. The representative day of each cluster is then used to build the POD basis. Various numbers of cluster have been tested: $k \in[1,35]$. For each cluster, the algorithm provides a centroid. The centroid is generally not a point from the data set. The closest day in the cluster data set is then designated as the representative day of the data set. The selection of the closest day is based on the surface temperature variable. Indeed, the latter is the key parameter of the model (it drives the surface energy balance of the soil). In order to select 
the closest day, the integral of the surface temperature of each day in the cluster is compared to the integral of the surface temperature of the centroids (see Figure 3). Figure 5 presents the evolution of the accuracy of the POD basis depending on the number of representative days considered in the training pe$\operatorname{riod} \Omega_{\text {out }}$. This result is given for various numbers of

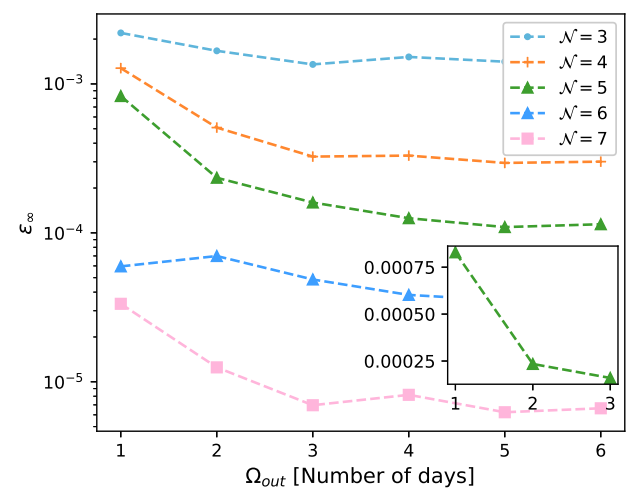

Figure 5: Influence of the number of representative days in the training period $\Omega_{\text {out }}$ on the POD basis accuracy using $k$-means clustering methodology.

modes $\mathcal{N}$ in the POD basis. The basis error decreases as the number of clusters increases. The previously described plateau is reached faster. For $\mathcal{N}=5$, an error of $\varepsilon=1.210^{-4}$ is reached with a $\Omega_{\text {out }}=3$ days. Thereby, a 3 days training period is enough to reach the desired accuracy.

Figure 6 depicts the clustering based on the surface temperature variable for 3 clusters. The output data set has been divided into three groups with seasonal characteristics: winter (in pink), summer (in green) and mid-season periods (in blue). This first study

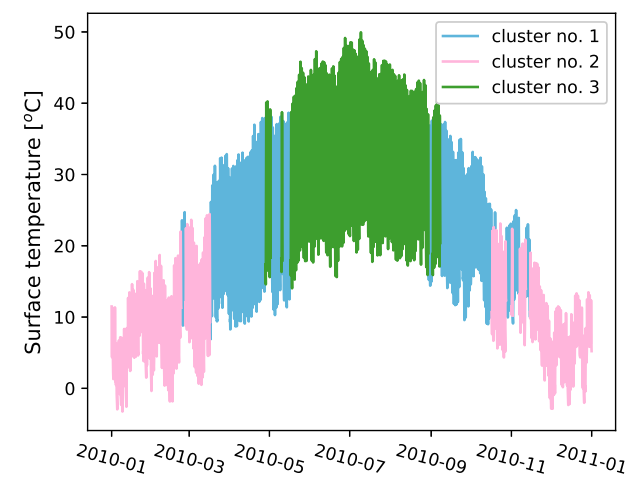

Figure 6: Clustering based on the surface temperature variable for 3 clusters.

carried out on the output data set has shown that the training period can be reduced to 3 days using the clustering method. The obtained clustering of the soil temperature is defined as the reference clustering in the following sections. The main objective now is to reduce the training climate, in order to reduce the LOM simulation period.

\section{From clustering the output data set to the in- put data set}

The first aim here is to verify if it is possible to obtain a similar training data set, by applying the clustering methodology to the input data set. For that purpose, the clustering methodology is applied to the boundary condition variables of the LOM. The method is applied on: (1) the air temperature $T_{a},(2)$ the air temperature and SW radiative heat flux through an equivalent temperature $T_{e, 1},(3)$ the air temperature, the SW and the LW radiative heat flux through an equivalent temperature $T_{e, 2}$. The equivalent temperatures are defined as follows:

$$
\begin{gathered}
T_{e, 1}=T_{a}+\frac{q_{S W}}{h} \\
T_{e, 2}=T_{a}+\frac{q_{S W}}{h}+\frac{q_{L W}}{h}
\end{gathered}
$$

The performance of each classification is compared to the reference classification (clustering performed on the output data set directly).

Different indicators can be used to assess the quality of the clustering method knowing its class (reference classification of each days). Three indicators are used here to give an information on how the days are classified compared to the reference classification. The mutual information (MI) score indicates the agreement of the two classifications obtained. The homogeneity verifies that each cluster contains only members of a single class, and the completeness verifies that all members of a given class are assigned to the same cluster. They are all bounded between 0 and 1,1 being the better score. The three first lines of table 1 present the results. The use of the equivalent temperatures instead of only the air temperature increases all the scores. The greatest the number of variable used to perform the clustering the better the days classification quality.

For each variable, the clustering methodology di-

Table 1: Clustering performance evaluation based on five criteria.

\begin{tabular}{|c|c|c|c|}
\hline & $T_{a}$ & $T_{e, 1}$ & $T_{e, 2}$ \\
\hline MI & 0.54 & 0.65 & 0.74 \\
\hline Homogeneity & 0.54 & 0.66 & 0.74 \\
\hline Completeness & 0.55 & 0.67 & 0.74 \\
\hline$\varepsilon_{\infty} \mathcal{N}=5$ & $1.210^{-6}$ & $1.110^{-6}$ & $1.910^{-6}$ \\
\hline
\end{tabular}

vides the input data set into 3 classes, for each class, a centroid is defined. As done previously, the closest day in the input data set is designated as the representative day. Finally the POD basis is built with the designated days. The arrangement of representative days in the training data set has no influence on the final POD basis accuracy.

Figure 7 presents the three training data sets obtained to build the POD basis. It compares dimensionless surface temperatures. For winter and summer the four days have similar characteristics. However, the mid-season days differ depending on the variable used to define the clusters. The use of the air temperature and SW radiative heat flux $T_{e, 1}$ gives the closest representative days. Last line of table 1 gives an information on the basis accuracy for five modes. The accuracy of each basis is in the same 

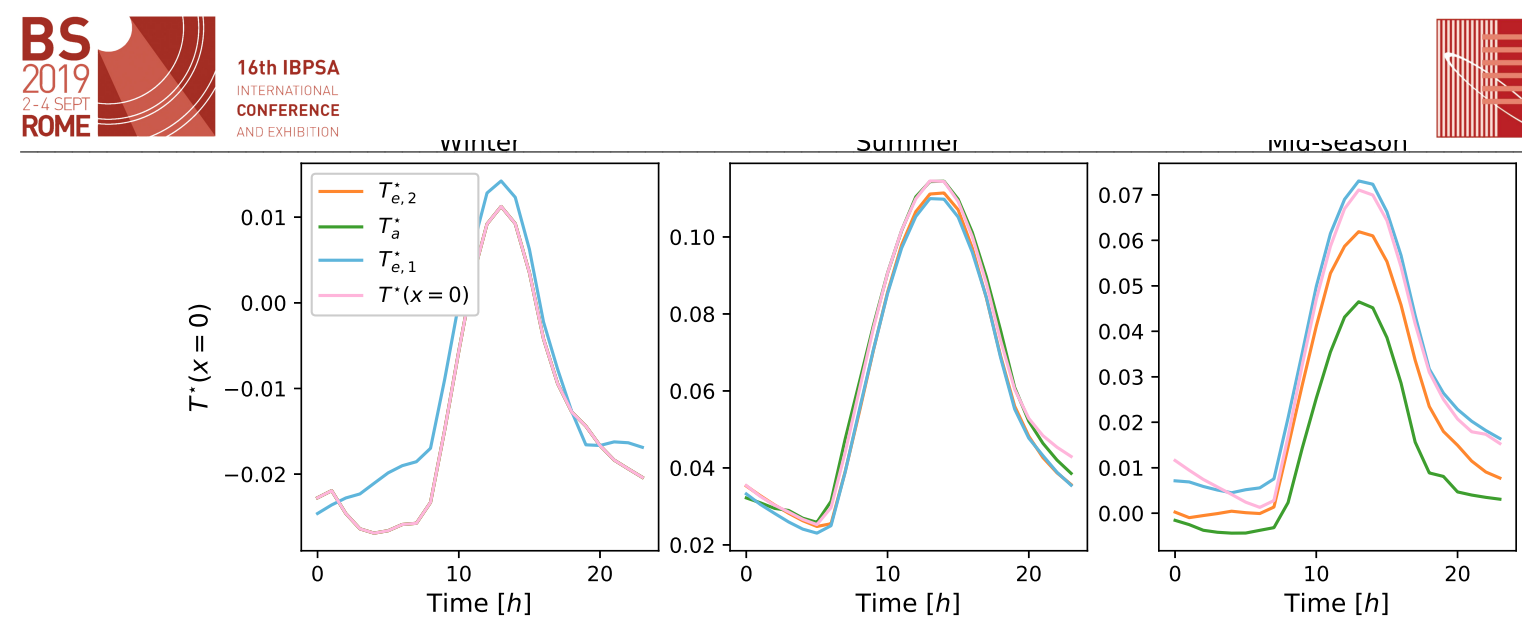

Figure 7: Dimensionless surface temperature of the representative days of each cluster.

order of magnitude than the error presented for the reference classification $\left(1.210^{-4}\right)$.

The use of the equivalent temperature with both SW and LW radiative heat flux increases the days' classification quality. However, in practice, the LW radiative heat flux is not available in the input data set as it is calculated by SOLENE-Microclimat at each time step. It is then more convenient to define the input training climate based on available data: air temperature and SW radiative heat flux.

\section{Building the input training climate}

It has been shown that for the specific case study, the POD basis can be built at least with a sequence of three representative days defined as the center of each class.

Each class and its center can be defined by clustering the output or the input data sets. As described below, in the case of a dynamical problem, the solution at a time step $t^{n}$ depends on the solution at the previous ones $t^{n-1}$ or $t^{n-2}$. If the LOM is run with a training climate of three days, the surface temperature obtained will have different characteristics than the one obtained with a full climate. The effect of inertia should then be taken into account to get a training climate that gives similar results.
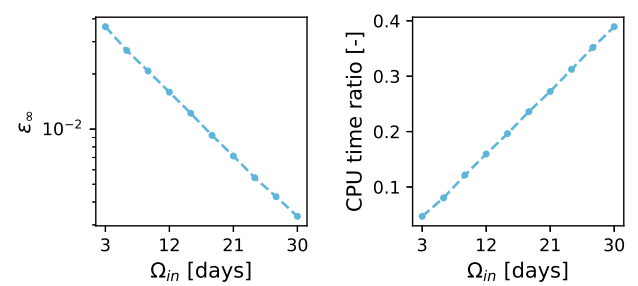

Figure 8: Evolution of the error and the CPU time calculation ratio between the LOM output for the training climate period $\Omega_{\text {in }}$ and for a full climate.

Thus, in order to take into account the inertia, several days are added to the training climate made of the representative days. Only days before the representative days are added to the training climate. The influence of the number of added days is studied through two factors: (1) the reproduction of the representative days characteristics in the output data set, (2) the final accuracy of the POD basis.
First, the LOM output for the three representative days are compared to a full training climate $\Omega_{t}$ and to a reduced training climate $\Omega_{i n}$. Figure 8 presents the evolution of the error depending on the training climate period $\Omega_{i n}$. For each point on the curve a supplementary day is added before each representative day. The error decreases as the number of days in the training climate increases. It is difficult to select an optimal number of days. Figure 8 also presents the evolution of the CPU calculation time ratio as a function of the number of days in the training climate $\Omega_{i n}$. The CPU calculation time increases linearly with the number of days.

Finally, the influence of the training climate period on the accuracy of the approximation basis $\Omega_{i n}$ is presented in figure 9 . The results are presented for several numbers of modes $\mathcal{N}$ of the POD basis. The error decreases as the training climate period increases. However it reaches a plateau. This plateau represents the accuracy of the POD basis. If the training climate has reached the basis accuracy (which is proportional to the number of modes in the basis), adding a supplementary day to the training climate will not improve the overall basis accuracy. An optimal number of days can then be defined. For example, for $\mathcal{N}=5$, adding 7 days before each representative day is enough to get an error of $1.010^{-4}$. It results in a training climate made of 24 days.

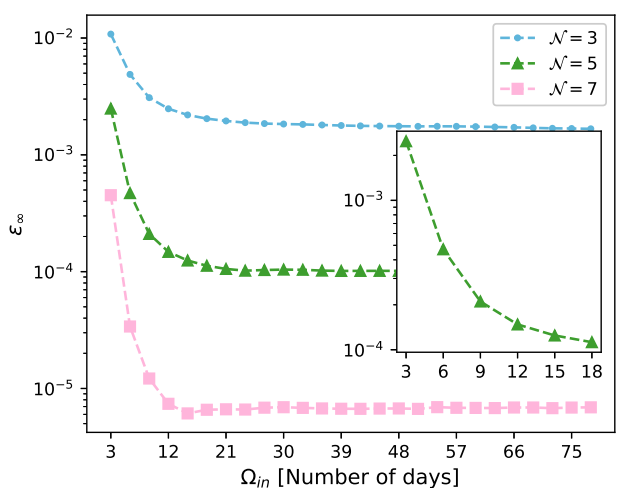

Figure 9: Evolution of the basis approximation error as a function of the training climate period $\Omega_{i n}$.

\section{Combined model performance}

The previously defined training climate is now evaluated to build the POD basis of the combined para- 
metric model. The performance of the training climate is assessed on the overall accuracy and computational time of the combined model. A parametric model using a POD basis built on the full climate data is compared to the one using the training climate. The temperature profiles of both combined ROM are compared to the LOM model for the entire simulation period. Both combined models are built using a POD basis with $\mathcal{N}=5$ and $\mathcal{M}=38$ PGD modes. The combined model with a full climate learning period has an error of $\varepsilon_{\infty}=0.0077$, while the one using a training climate of 24 days has an error of $\varepsilon_{\infty}=0.0080$. The use of the training climate methodology increases the model dimensionless error. However, this error decreases with the number of days in the training climate. For 24 days, the error obtained are in the same order of magnitude. The offline computation time are depicted in table 2 .

Table 2: Offline CPU calculation time ratio.

\begin{tabular}{|c|c|c|}
\hline$\Omega_{\text {in }}$ [days] & 365 & 24 \\
\hline LOM & 1.000 & 0.054 \\
\hline Building POD basis & 1.000 & 0.209 \\
\hline Total offline & 1.000 & 0.056 \\
\hline
\end{tabular}

The use of the training climate reduces drastically the offline computation time: $94.6 \%$ for the LOM calculation time and $79 \%$ for building the POD basis. The total global offline computation time reduction is by $94.4 \%$.

\section{Conclusion}

The k-means clustering method has been evaluated to build a short and representative training period for a POD basis. The full climate has been reduced to a training climate made of 3 representative days and a number of previous days to take the inertia into account. The overall accuracy of the model increases as the number of days included in the training climate increases. With an optimized training climate of 24 days ( 3 representative days and 21 days for the inertia), the overall combined accuracy of the model remains in the same order of magnitude $\varepsilon_{\infty}=0.008$ for a reduction of the offline computation time of $94.4 \%$. It contributes to the global improvement of the performance of the ROM combined model. Those results are encouraging. The replicability of the method should be tested on other meteorological data set, and soil composition. This paper focuses on urban soil behavior. However, building modeling is another challenge. A main question should be addressed in future work: How can we apply this methodology to building walls in a complex urban environment (shading effect, etc.) ?

\section{References}

Azam, M.-H., S. Guernouti, M. Musy, J. Berger, P. Poullain, and A. Rodler (2018). A mixed podpgd approach to parametric thermal impervious soil modeling: Application to canyon streets. Sustainable Cities and Society 42, 444-461.

Berger, J., W. Mazuroski, R. C. Oliveira, and N. Mendes (2018). Intelligent co-simulation: neural network vs. proper orthogonal decomposition applied to a 2 d diffusive problem. Journal of Building Performance Simulation, 1-20.

Borzacchiello, D., J. V. Aguado, and F. Chinesta (2017). Non-intrusive sparse subspace learning for parametrized problems. Archives of Computational Methods in Engineering, 1-24.

Chinesta, F., R. Keunings, and A. Leygue (2013). The proper generalized decomposition for advanced numerical simulations: a primer. Springer Science \& Business Media.

Domínguez-Muñoz, F., J. M. Cejudo-López, A. Carrillo-Andrés, and M. Gallardo-Salazar (2011). Selection of typical demand days for chp optimization. Energy and buildings 43(11), 3036-3043.

Fazlollahi, S., S. L. Bungener, P. Mandel, G. Becker, and F. Maréchal (2014). Multi-objectives, multiperiod optimization of district energy systems: I. selection of typical operating periods. Computers 8 Chemical Engineering 65, 54-66.

González, D., F. Masson, F. Poulhaon, A. Leygue, E. Cueto, and F. Chinesta (2012). Proper generalized decomposition based dynamic data driven inverse identification. Mathematics and Computers in Simulation 82(9), 1677-1695.

Mirzaei, P. A. (2015). Recent challenges in modeling of urban heat island. Sustainable Cities and Society 19, 200-206.

Musy, M., L. Malys, B. Morille, and C. Inard (2015). The use of solene-microclimat model to assess adaptation strategies at the district scale. Urban Climate 14, 213-223.

Oke, T. R. (2002). Boundary layer climates. Routledge.

Pedregosa, F., G. Varoquaux, A. Gramfort, V. Michel, B. Thirion, O. Grisel, M. Blondel, P. Prettenhofer, R. Weiss, V. Dubourg, J. Vanderplas, A. Passos, D. Cournapeau, M. Brucher, M. Perrot, and E. Duchesnay (2011). Scikit-learn: Machine learning in Python. Journal of Machine Learning Research 12, 2825-2830.

Ribault, C. (2017). Méthode d'optimisation multicrière pour l'aide à la conception des projets de densification urbaine. Ph. D. thesis, INSA Lyon. 